\title{
Klotho expression and nodal involvement as predictive factors for large cell lung carcinoma
}

\author{
Barbara Brominska ${ }^{1}$, Piotr Gabryel², Donata Jarmołowska-Jurczyszyn ${ }^{3}$, \\ Małgorzata Janicka-Jedyńska³, Andrzej Kluk³ , Maciej Trojanowski ${ }^{4}$, Beata Brajer-Luftmann5, \\ Kosma Woliński ${ }^{1}$, Rafał Czepczyński ${ }^{1}$, Paweł Gut ${ }^{1}$, Gabriel Bromiński ${ }^{1}$, Przemysław Majewski ${ }^{3}$, \\ Wojciech Dyszkiewicz², Marek Ruchała ${ }^{1}$
}

\author{
${ }^{1}$ Department of Endocrinology, Metabolism and Internal Medicine, Poznan University \\ of Medical Sciences, Poznan, Poland \\ 2Department of Thoracic Surgery, Poznan University of Medical Sciences, Poznan, \\ Poland \\ ${ }^{3}$ Department of Clinical Pathology, Poznan University of Medical Sciences, Poznan, \\ Poland \\ ${ }^{4}$ Department of Epidemiology and Cancer Prevention, Greater Poland Cancer Center, \\ Poznan, Poland \\ ${ }^{5}$ Department of Pulmonology, Allergology and Respiratory Oncology, \\ Poznan University of Medical Sciences, Poznan, Poland
}

Submitted: 18 October 2016

Accepted: 22 May 2017

Arch Med Sci 2019; 15 (4): 1010-1016

DOI: https://doi.org/10.5114/aoms.2018.75889

Copyright (c) 2018 Termedia \& Banach

\section{Abstract}

Introduction: Klotho has been recently described as a carcinogenesis suppressor. Large cell neuroendocrine lung carcinoma (LCNEC) is a rare, highly malignant neoplasm. In the light of increasing incidence of neuroendocrine tumours, biomarkers predicting survival are needed. We consider that Klotho might be one.

Material and methods: We analysed records of all patients diagnosed with LCNEC, atypical carcinoid and typical carcinoid operated on in our institution between 2007 and 2015. Initially, we found 134 cases. Forty-six specimens were unattainable and thus excluded from research. All patients diagnosed with LCNEC according to the WHO classification were included in the study. Immunohistochemical staining for Klotho was performed. We retrospectively reviewed patient charts and analysed multiple variables.

Results: Positive staining for Klotho was present in 36 tissue specimens, while 12 patients were Klotho-negative. Survival length was significantly higher in Klotho-positive cases ( $p=0.024)$, while advanced nodal status (N1 and N2) represented a marker of poor outcome $(p=0.011)$. In multivariate analysis, both Klotho presence $(p=0.015 ; \mathrm{HR}=0.37 ; 95 \% \mathrm{Cl}: 0.17-0.86)$ and nodal involvement ( $p=0.007 ; \mathrm{HR}=3.04 ; 95 \% \mathrm{Cl}: 1.37-6.82$ ) were independent prognostic factors. Tumour vessel invasion and visceral pleura infiltration were not associated with worse treatment results. Klotho presence predicted a favourable prognosis in these groups ( $p=0.018 ; p=0.007$ ) Conclusions: Our results suggest that Klotho might be a positive factor for predicting survival in LCNEC and nodal involvement a negative one. Thus, these two markers may assist in the selection of subjects with unfavourable prognosis and to personalise therapy regimens.

Key words: Klotho, large cell neuroendocrine lung carcinoma, survival, neuroendocrine, lung.

\author{
Corresponding author: \\ Barbara Brominska MD \\ Department \\ of Endocrinology, \\ Metabolism \\ and Internal Medicine \\ Poznan University \\ of Medical Sciences \\ 49 Przybyszewskiego St \\ 60-355 Poznan, Poland \\ Phone: +486186913 30 \\ Fax: +48 618691682 \\ E-mail: barbarabrominska@ \\ gmail.com
}




\section{Introduction}

Klotho was first discovered as an anti-ageing gene. It encodes a single-pass transmembrane protein, which is involved in multiple biological processes. In soluble form, secreted into the circulation, it participates in the regulation of ion channels and growth factor levels and increases resistance to oxidative stress $[1,2]$. The membrane part creates a complex with fibroblast growth factor (FGF) receptors and functions as a co-receptor for FGF23 - bone-derived hormone - useful in calcium-phosphate metabolism [3, 4].

Recently, Klotho has been described as a marker for cancer. Aberrant Klotho expression has been found in several types of neoplasm, namely breast [5], hepatocellular [6], thyroid [7], and colorectal cancer [8]. Downregulation and epigenetic modulation of Klotho contribute to dysregulation of gene expression, resulting in reduced apoptosis and increased proliferation of neoplasm cells. Klotho influences the insulin-growth factor (IGF), FGF and Wnt signalling pathways [9]. Although it mostly acts as a tumour suppressor, this protein functions as an oncogene in epithelial ovarian cancer [10].

The role of Klotho has been studied in lung cancers, where it depresses proliferation and induces apoptosis of tumours [11]. Klotho downregulation is involved in chemotherapy resistance; thus, it might be a future therapeutic goal [12]. Klotho has been shown to be a good predictor of survival in patients with resected small cell lung cancer (SCLC) [13].

Large cell neuroendocrine lung carcinoma (LCNEC) is a rare, highly malignant neoplasm accounting for $2-3 \%$ of lung cancers. The 5 -year survival rate in post-operative patients varies from $20 \%$ to $55 \%$, which is similar to SCLC. Recurrence and distant metastases occur very early, even if resection is complete and in early stages. There are no equivocal treatment regimens, so management is individualised by multidisciplinary teams [14]. Moreover, lung cancer is relatively asymptomatic at the beginning, which results in delayed diagnosis [15]. In the light of the increasing incidence of neuroendocrine tumours [16], biomarkers predicting survival and recurrence and those associated with distant metastases are needed. We consider that the Klotho protein, due to its role in tumourigenesis, might be one such biomarker. In this study, we examined Klotho expression in LCNEC in relation to survival rates and other parameters.

\section{Material and methods}

We analysed records of all patients diagnosed with LCNEC, atypical carcinoid and typical carcinoid operated on in our institution between 2007 and 2015. Initially, we found 134 cases. Forty-six specimens were unattainable and thus excluded from research. All patients diagnosed with LCNEC according to the WHO classification were included in the study. Immunohistochemical staining was performed for chromogranin, synaptophysin and NCAM. This was done routinely at the time of diagnosis, using standard methods.

Formalin-fixed, paraffin wax embedded tissue specimens were cut into $4-\mu \mathrm{m}$ sections and mounted on SuperFrost Plus adhesive microscope slides (Menzel Gläser). Deparaffinisation, hydration and heat-induced epitope retrieval were carried out by cooking in low pH Dako EnVision FLEX Target Retrieval Solution (Glostrup, Denmark) for 50 min at $97^{\circ} \mathrm{C}$ in a water bath. Endogenous peroxidase was blocked with Dako EnVision FLEX Peroxidase-Blocking Reagent (Glostrup, Denmark). Next, sections were incubated in Leica Biosystems Novocastra Protein Block (Wetzlar, Germany) for $10 \mathrm{~min}$. Then, tissue specimens were incubated overnight with monoclonal mouse Anti-Human Ki67 Antigen antibody (Dako) (dilution 1 : 500), and rabbit monoclonal antibody (EPR6856) to Klotho (Abcam) (dilution 1 : 2000) in a humid chamber at $4^{\circ} \mathrm{C}$. The primary antibody was diluted in Dako EnVision FLEX Antibody Diluent (Glostrup, Denmark). Immunodetection was achieved using the Novolink Polymer Detection System (Leica Biosystems), which is a two-step streptavidin-biotinperoxidase method. Visualisation was achieved using 3,3'-diaminobenzidine tetrachloride (DAB, Leica Microsystems). Sections were counterstained with Mayer's haematoxylin, dehydrated, cleared, and mounted in DPX. Immunohistochemical staining was evaluated by two experienced pathologists working in the Department of Clinical Pathology in Poznan. Staining for Klotho was considered positive when more than $10 \%$ of tumour cells were stained, as in previous studies [13, 17].

TNM staging was determined according to the $7^{\text {th }}$ edition of the TNM classification. We retrospectively reviewed patient charts and analysed multiple variables in January 2015 (patients included were operated on between June 2008 and March 2014). Additionally, survival analysis of the patient cohort was conducted. We reviewed survival data in March 2016. The study design was accepted by our institution's bioethical committee. All procedures performed in studies involving human participants were in accordance with the ethical standards of the institutional and/or national research committee and with the 1964 Helsinki Declaration and its later amendments or comparable ethical standards.

\section{Statistical analysis}

Statistical analysis was performed using Statistica version 10 (StatSoft Inc., Tulsa, U.S.A.) 
B. Brominska, P. Gabryel, D. Jarmołowska-Jurczyszyn, M. Janicka-Jedyńska, A. Kluk, M. Trojanowski, B. Brajer-Luftmann, K. Woliński, R. Czepczyński, P. Gut, G. Bromiński, P. Majewski, W. Dyszkiewicz, M. Ruchała

Continuous variables were given as median, minimal and maximal values. Categorical variables were presented as numbers (\%). The level of statistical significance was set at the $p$-value of 0.05 . Values over 0.05 were considered not significant. We defined overall survival as the time from the operation to the end-point of the study (either death or end of the observation). Cumulative survival was assessed with the Kaplan-Meier method. Differences between survival rates in selected groups were evaluated with the log-rank test. We used the Cox regression model to examine the effects of selected features on survival. Variables were considered significant in univariate analyses. In the final model we included: Klotho expression, lymph node involvement (entry level: 0.05) and tumour vessel invasion. The latter was included due to its vital role in neoplasm progression.

\section{Results}

Clinical and laboratory data of the study groups are shown in Table I. Video-assisted thoracoscopic surgery (VATS) was performed in 12 cases, thoracotomy in 36. Mean follow-up time was 20.7 months (from 0.6 to 67.5 ) (Figure 1). Positive staining for Klotho was present in 36 (75\%) tissue specimens, while 12 (25\%) patients were Klotho-negative. At the time of follow-up, 18 Klotho-positive patients were alive, while 18 died. In the Klotho-negative part, only 2 were alive, while 10 died. The median follow-up survival for the Klotho-positive group was 22.6, while for the negative group it was 15.2 months. Next, we compared these two groups in terms of survival length, which was significantly higher in Klotho-positive cases ( $p=0.024$; Figure 2$)$. Moreover, advanced nodal status (N1 and N2) represented a marker of poor outcome $(p=0.011$; Figure 3 ). We used the Cox regression model to examine the effects of specific features on survival (independent variables: Klotho expression, nodal involvement, tumour vessel invasion; de-

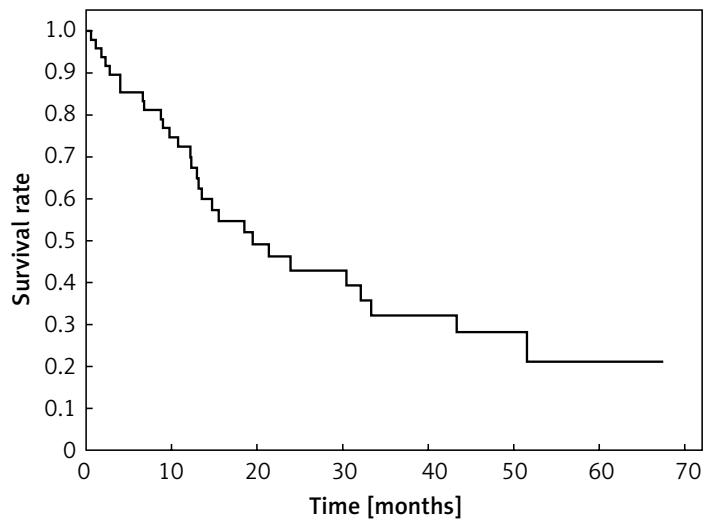

Figure 1. Overall survival in patients with LCNEC, Kaplan-Meier method
Table I. Characteristics of patients with LCNEC

\begin{tabular}{|c|c|}
\hline Parameter & Results \\
\hline \multicolumn{2}{|l|}{ Gender: } \\
\hline Female & $17(35 \%)$ \\
\hline Male & $31(65 \%)$ \\
\hline Age, mean (range) [years] & $63.5(48-78)$ \\
\hline Size of the tumour, mean (range) $[\mathrm{mm}]$ & $40.6(10-90)$ \\
\hline Ki-67, mean (range) (\%) & $37(10-90)$ \\
\hline $\begin{array}{l}\text { Time of observation, mean (range) } \\
\text { [months] }\end{array}$ & $20.7(0.6-67.5)$ \\
\hline \multicolumn{2}{|l|}{ Type of operation, $n$ (\%): } \\
\hline Lobectomy & $41(85)$ \\
\hline Pneumonectomy & $5(10)$ \\
\hline Wedge resection & $1(2.5)$ \\
\hline Segment resection & $1(2.5)$ \\
\hline \multicolumn{2}{|l|}{ TNM classification, $n(\%)$ : } \\
\hline Class la & $6(12.5)$ \\
\hline Class Ib & $9(19)$ \\
\hline Class Ila & $18(37.5)$ \\
\hline Class IIb & $4(8)$ \\
\hline Class IIIa & 9 (19) \\
\hline Class IV & $2(4)$ \\
\hline \multicolumn{2}{|l|}{ Visceral pleura infiltration, $n(\%)$ : } \\
\hline Yes & $16(33)$ \\
\hline No & $32(67)$ \\
\hline \multicolumn{2}{|l|}{ Parietal pleura infiltration, $n(\%)$ : } \\
\hline Yes & $4(8)$ \\
\hline No & $44(92)$ \\
\hline \multicolumn{2}{|l|}{ Tumour vessel invasion, $n(\%)$ : } \\
\hline Yes & $11(23)$ \\
\hline No & $37(77)$ \\
\hline \multicolumn{2}{|l|}{ Lymph node involvement, $n(\%)$ : } \\
\hline Yes & $23(48)$ \\
\hline No & $25(52)$ \\
\hline \multicolumn{2}{|l|}{ Klotho presence, $n(\%)$ : } \\
\hline Yes & $36(75)$ \\
\hline No & $12(25)$ \\
\hline Alive at time of analysis, $n(\%)$ & $15(31)$ \\
\hline Cancer-related death, $n(\%)$ & $31(65)$ \\
\hline Other causes of death, $n(\%)$ & $2(4)$ \\
\hline
\end{tabular}




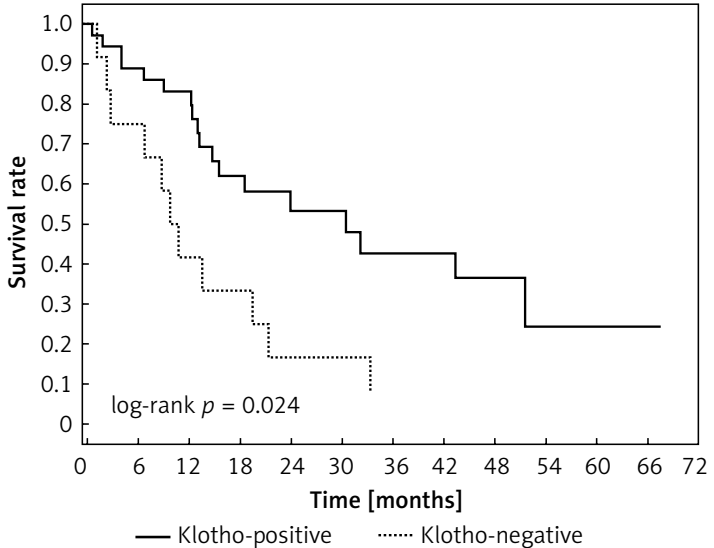

Figure 2. Kaplan-Meier method showing overall survival in Klotho-positive and -negative patients

pendent variables: survival). Under multivariate analysis, both Klotho presence $(p=0.015 ; \mathrm{HR}=$ 0.37; confidence interval 95\%: $0.17-0.86)$ and nodal involvement $(p=0.007 ; \mathrm{HR}=3.04$; confidence interval 95\%: 1.37-6.82) were independent prognostic factors, while presence of tumour vessel invasion did not affect the outcome $(p=0.094$; $H R=0.44$; confidence interval 95\%: 0.16-1.18) (Table II). We also assessed whether Klotho influenced the prognosis in patients with and without nodal metastases. We did not find any connection between Klotho and survival times in subjects either with $(p=0.084)$ (Figure 4$)$ or without nodal involvement $(p=0.55)$. As mentioned above, tumour vessel invasion was reported in 11 cases. During the observation period, 6 of these patients died ( 5 of them were Klotho-negative), and 5 were still alive (all Klotho-positive). Tumour vessel invasion was not associated with shorter survival $(p=0.616)$. However, Klotho presence was predictive of a favourable prognosis in patients who had initially presented with tumour vessel invasion $(p=0.018$; Figure 5). Neither visceral $(p=0.35)$ nor parietal pleura $(p=-0.89)$ invasion was a prognostic marker. Klotho expression was associated with better outcome in the group with visceral pleura infiltration ( $p=0.007$ ) (Figure 6), but not in the group without $(p=0.14)$.

Table II. Effects of specific features on survival (multivariate analysis). Cox regression model

\begin{tabular}{|lll|}
\hline Variable & HR $(95 \% \mathrm{Cl})$ & $P$-value \\
\hline $\begin{array}{l}\text { Lymph node metastases: } \\
\text { Yes vs. No }\end{array}$ & $3.04(1.37-6.82)$ & 0.0066 \\
\hline $\begin{array}{l}\text { Klotho presence: } \\
\text { Yes vs. No }\end{array}$ & $0.37(0.17-0.86)$ & 0.0149 \\
\hline $\begin{array}{l}\text { Tumour vessel invasion: } \\
\text { Yes vs. No }\end{array}$ & $0.44(0.16-1.18)$ & 0.1031 \\
\hline
\end{tabular}

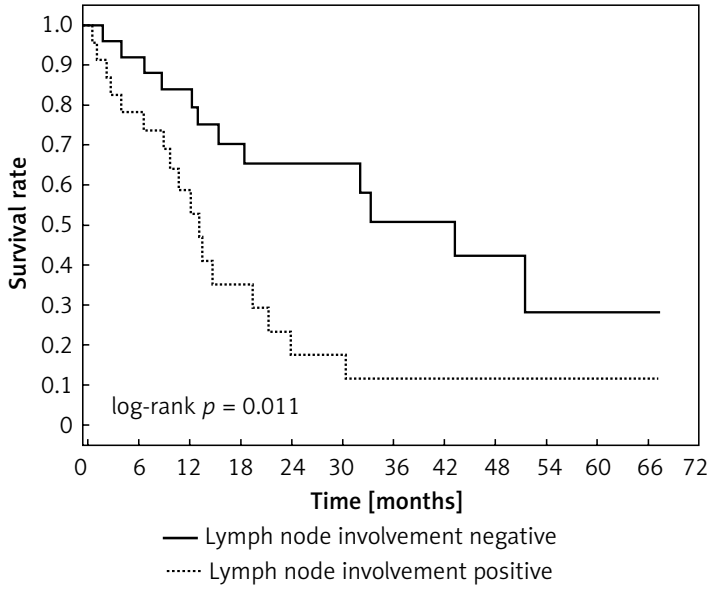

Figure 3. Kaplan-Meier plot depicting differences in overall survival between patients with and without nodule involvement

\section{Discussion}

Large cell neuroendocrine lung carcinoma is a rare and aggressive tumour with very poor prognosis. Surgery is a first-line treatment. The role of pre-operative and adjuvant chemotherapy is not well established [18-21]. Patients usually present with non-specific symptoms. Occasionally, the tumour may cause carcinoid, Cushing syndrome or acromegaly [22-24]. Most data concerning this entity derive from small retrospective studies, and there are no ongoing prospective clinical trials concerning the best treatment options [25]. In this setting, there is an urgent need to define biomarkers predictive of survival and therapeutic efficacy.

Klotho, an anti-senescence gene, is a potential tumour suppressor in lung cancer. Due to the involvement of Klotho in carcinogenesis, we assessed the role of this protein in LCNEC. Although it was previously shown that Klotho might be associated with longer survival in LCNEC, the small

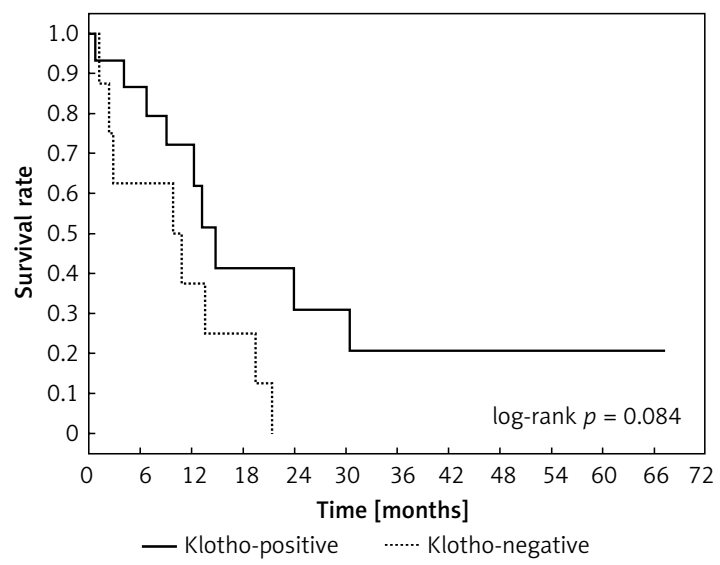

Figure 4. Kaplan-Meier plot showing differences in survival in patients in Klotho-positive and -negative subgroups among subjects with lymph node metastases 
B. Brominska, P. Gabryel, D. Jarmołowska-Jurczyszyn, M. Janicka-Jedyńska, A. Kluk, M. Trojanowski, B. Brajer-Luftmann, K. Woliński, R. Czepczyński, P. Gut, G. Bromiński, P. Majewski, W. Dyszkiewicz, M. Ruchała

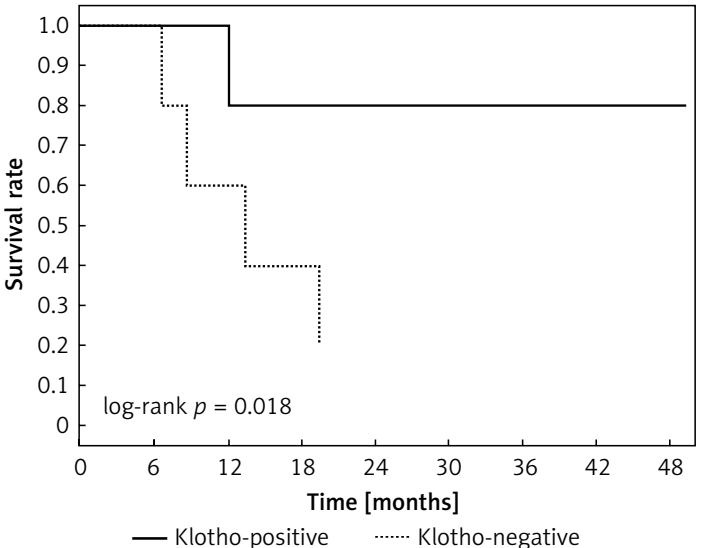

Figure 5. Kaplan-Meier plot showing differences in survival in patients in Klotho-positive and -negative subgroups among subjects with tumour vessel invasion

study sample limited the usefulness of the data [17]. Susceptibility to various cancers and disease activity may be to some extent associated with genetic differences among ethnic groups [26]. Thus, we aim to provide insight into our population. Our results suggest that Klotho might be an independent factor for predicting favourable outcome in LCNEC. Klotho's effect on neoplasm behaviour may be mediated by IGF [27], FGF [28, 29] and Wnt signalling pathways. Klotho overexpression is associated with decreased and silencing with increased lung cancer cell line growth (A549). High transmembrane and secreted Klotho expression inhibits motility in those cell lines [11, 30]. Moreover, Klotho attenuates resistance to chemotherapy [12]. Considering those data and our results, Klotho could possibly serve as a useful marker to stratify high-risk patients who require additional treatment.

We also demonstrated that lymph node involvement independently, negatively influences prognosis in LCNEC, which is in line with results from previous studies [31-33]. Furthermore, we investigated whether Klotho expression impacts

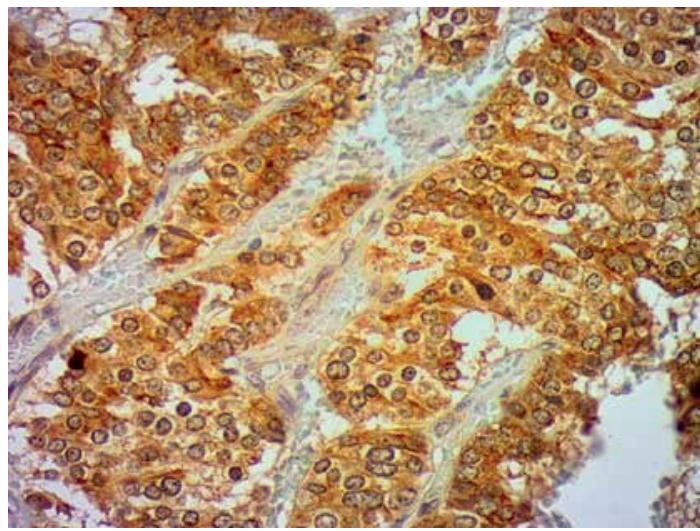

Figure 7. Klotho expression. Immunohistochemical staining $(400 x)$

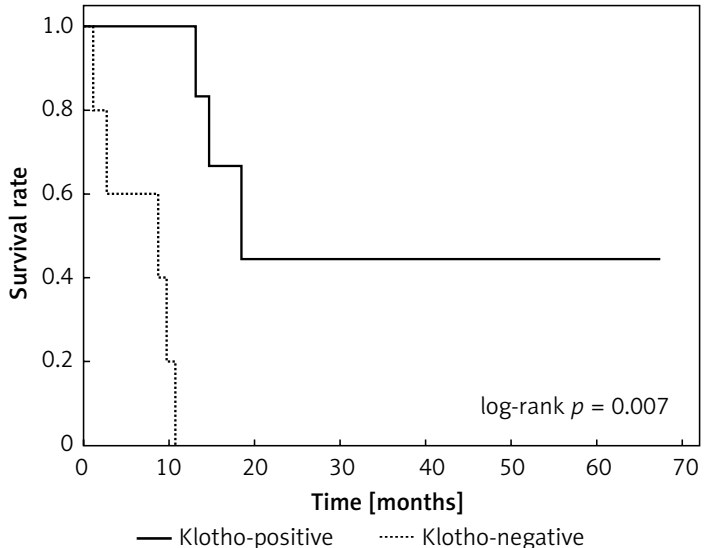

Figure 6. Kaplan-Meier plot showing differences in survival in patients in Klotho-positive and -negative subgroups among subjects with visceral pleura invasion

survival in patients with or without lymph node metastasis. Our research did not show differences in outcomes in either Klotho-negative or -positive subgroups. Conversely, Usuda et al. demonstrated that in patients without lymph node involvement Klotho improves the outcome, but lymph node status alone was not an independent prognostic factor [17]. These differences might result from the study sample size.

There have been several studies concerning tumour vessel invasion as a prognostic factor in LCNEC, but the results were inconclusive [32, 34]. Although in our research it was not associated with the outcome, we decided to perform further analysis. We disclosed that patients with tumour vessel invasion benefited in terms of survival from Klotho expression. Moreover, Klotho was associated with better outcome in patients without tumour vessel invasion in a study by Usuda et al. [17]. Therefore, we presume that Klotho might be useful to select lower-risk patients, with reference to tumour vessel invasion.

Visceral pleura infiltration is known as an indicator of aggressiveness of non-small cell lung

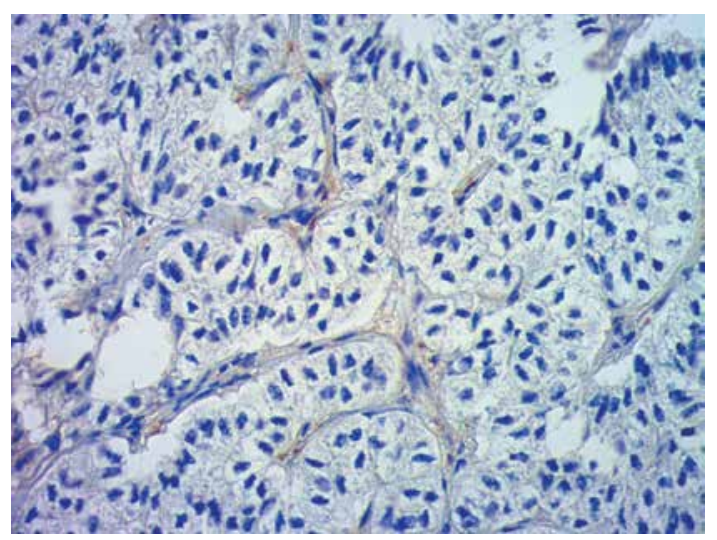

Figure 8. Negative reaction. Immunohistochemical staining (400x) 
cancer (NSCLC). It is usually associated with extensive nodal involvement [35]. Possibly, exfoliated tumour cells are drained from the pleural cavity through lymphatic vessels to the bloodstream, resulting in systemic neoplasm dissemination [36]. Although numerous studies focus on NSCLC, data concerning neuroendocrine tumours are limited. There was a single study by Grand et al., in which surgical characteristics of high-grade lung tumours and large cell carcinoma were compared. Visceral pleura invasion was most common in LCNEC combined with NSCLC. The authors did not study the association between visceral invasion and survival data [37]. We disclosed that visceral invasion was not associated with poorer prognosis in LCNEC. Moreover, Klotho expression in this group predicted favourable prognosis.

Our study was conducted at a single institution on a relatively small sample, which might be a major limitation. It must be remembered, however, that collecting a larger cohort is difficult since the incidence of LCNEC is low (between $2.1 \%$ and $3.5 \%$ of all lung cancer cases) [38]. Nevertheless, multi-institutional analysis is vital for any potential biomarker to be implemented into routine procedures.

In conclusion, we found that both Klotho and lymph node involvement might be independent prognostic factors. While Klotho expression was associated with favourable outcome, lymph node metastases predicted worse survival. Moreover, we analysed the impact of Klotho expression on survival with reference to lymph node involvement, tumour vessel invasion and pleural infiltration. As the results are promising, Klotho may assist in the future in selection of subjects with favourable prognosis and in the personalisation of therapy regimens. However its prognostic significance requires further studies (Figures 7,8 ).

\section{Conflict of interest}

The authors declare no conflict of interest.

\section{References}

1. Kim JH, Hwang KH, Park KS, Kong ID, Cha SK. Biological role of anti-aging protein Klotho. J Lifestyle Med 2015; 5: $1-6$.

2. Dąbrowska AM, Tarach JS. Soluble Klotho protein as a novel serum biomarker in patients with acromegaly. Arch Med Sci 2014; 12: 222-6.

3. Kuro-o M. Klotho and aging. Biochim Biophys Acta 2009; 1790: 1049-58.

4. Moor MB, Bonny O. Ways of calcium reabsorption in the kidney. Am J Physiol Renal Physiol 2016; 310: F1337-50.

5. Trost N, Pena-Llopis S, Koirala S, et al. gammaKlotho is a novel marker and cell survival factor in a subset of triple negative breast cancers. Oncotarget 2016; 7 : 2611-28.

6. Sun H, Gao Y, Lu K, et al. Overexpression of Klotho suppresses liver cancer progression and induces cell apop- tosis by negatively regulating wnt/beta-catenin signaling pathway. World I Surg Oncol 2015; 13: 307.

7. Dai D, Wang O, Li X, Liu J, Ma X, Xu W. Klotho inhibits human follicular thyroid cancer cell growth and promotes apoptosis through regulation of the expression of stanniocalcin-1. Oncol Rep 2016; 35: 552-8.

8. Liu C, Cui W, Wang L, et al. Klotho gene polymorphisms are related to colorectal cancer susceptibility. Int J Clin Exp Pathol 2015; 8: 7446-9.

9. Zhou X, Wang X. Klotho: a novel biomarker for cancer. J Cancer Res Clin Oncol 2015; 141: 961-9.

10. Lu L, Katsaros D, Wiley A, de la Longrais IA, Puopolo M, $\mathrm{Yu} \mathrm{H}$. Klotho expression in epithelial ovarian cancer and its association with insulin-like growth factors and disease progression. Cancer Invest 2008; 26: 185-92.

11. Chen B, Wang X, Zhao W, Wu J. Klotho inhibits growth and promotes apoptosis in human lung cancer cell line A549. J Exp Clin Cancer Res 2010; 29: 99.

12. Wang Y, Chen L, Huang G, et al. Klotho sensitizes human lung cancer cell line to cisplatin via PI3k/Akt pathway. PLoS One 2013; 8: e57391.

13. Usuda J, Ichinose S, Ishizumi T, et al. Klotho predicts good clinical outcome in patients with limited-disease small cell lung cancer who received surgery. Lung Cancer 2011; 74: 332-7.

14. Filosso PL, Ferolla P, Guerrera F, et al. Multidisciplinary management of advanced lung neuroendocrine tumors. J Thorac Dis 2015; 7 (Suppl 2): S163-71.

15. Sharma D, Newman TG, Aronow WS. Lung cancer screening: history, current perspectives, and future directions. Arch Med Sci 2015; 11: 1033-43.

16. Hallet J, Law CH, Cukier M, Saskin R, Liu N, Singh S. Exploring the rising incidence of neuroendocrine tumors: a population-based analysis of epidemiology, metastatic presentation, and outcomes. Cancer 2015; 121: 589-97.

17. Usuda J, Ichinose S, Ishizumi T, et al. Klotho is a novel biomarker for good survival in resected large cell neuroendocrine carcinoma of the lung. Lung Cancer 2011; 72: 355-9.

18. Fasano M, Della Corte CM, Papaccio F, Ciardiello F, Morgillo F. Pulmonary large-cell neuroendocrine carcinoma: from epidemiology to therapy. J Thorac Oncol 2015; 10: 1133-41.

19. Iyoda A, Hiroshima K, Moriya $\mathrm{Y}$, et al. Postoperative recurrence and the role of adjuvant chemotherapy in patients with pulmonary large-cell neuroendocrine carcinoma. J Thorac Cardiovasc Surg 2009; 138: 446-53.

20. Lo Russo G, Pusceddu S, Proto C, et al. Treatment of lung large cell neuroendocrine carcinoma. Tumour Biol 2016; 37: 7047-57.

21. Liu KJ, Guan ZZ, Liang Y, et al. A double-blind, randomized phase II study of dicycloplatin plus paclitaxel versus carboplatin plus paclitaxel as first-line therapy for patients with advanced non-small-cell lung cancers. Arch Med Sci 2014; 10: 717-24.

22. Ghazi AA, Abbasi Dezfooli A, Amirbaigloo A, et al. Ectopic Cushing's syndrome secondary to lung and mediastinal tumours: report from a tertiary care centre in Iran. Endokrynol Pol 2015; 66: 2-9.

23. Gut P, Waligórska-Stachura J, Czarnywojtek A, et al. Management of the hormonal syndrome of neuroendocrine tumors. Arch Med Sci 2017; 13: 515-24.

24. Gut P, Czarnywojtek A, Fischbach J, et al. Chromogranin A - unspecific neuroendocrine marker. Clinical utility and potential diagnostic pitfalls. Arch Med Sci 2016; 12: 1-9.

25. Glisson BS, Moran CA. Large-cell neuroendocrine carcinoma: controversies in diagnosis and treatment. J Natl Compr Canc Netw 2011; 9: 1122-9. 
B. Brominska, P. Gabryel, D. Jarmołowska-Jurczyszyn, M. Janicka-Jedyńska, A. Kluk, M. Trojanowski, B. Brajer-Luftmann, K. Woliński, R. Czepczyński, P. Gut, G. Bromiński, P. Majewski, W. Dyszkiewicz, M. Ruchała

26. Henderson BE, Lee NH, Seewaldt V, Shen $\mathrm{H}$. The influence of race and ethnicity on the biology of cancer. Nat Rev Cancer 2012; 12: 648-53.

27. Abramovitz L, Rubinek T, Ligumsky H, et al. KL1 internal repeat mediates Klotho tumor suppressor activities and inhibits bFGF and IGF-I signaling in pancreatic cancer. Clin Cancer Res 2011; 17: 4254-66.

28. Feng S, Dakhova O, Creighton CJ, Ittmann M. Endocrine fibroblast growth factor FGF19 promotes prostate cancer progression. Cancer Res 2013; 73: 2551-62.

29. Poh W, Wong W, Ong H, et al. Klotho-beta overexpression as a novel target for suppressing proliferation and fibroblast growth factor receptor-4 signaling in hepatocellular carcinoma. Mol Cancer 2012; 11: 14.

30. Chen B, Ma X, Liu S, Zhao W, Wu J. Inhibition of lung cancer cells growth, motility and induction of apoptosis by Klotho, a novel secreted Wnt antagonist, in a dose-dependent manner. Cancer Biol Ther 2012; 13: 1221-8.

31. Eichhorn F, Dienemann H, Muley T, Warth A, Hoffmann H. Predictors of survival after operation among patients with large cell neuroendocrine carcinoma of the lung. Ann Thorac Surg 2015; 99: 983-9.

32. Doddoli C, Barlesi F, Chetaille B, et al. Large cell neu roendocrine carcinoma of the lung: an aggressive disease potentially treatable with surgery. Ann Thorac Surg 2004; 77: 1168-72.

33. Paci M, Cavazza A, Annessi V, et al. Large cell neuroendocrine carcinoma of the lung: a 10-year clinicopathologic retrospective study. Ann Thorac Surg 2004; 77: 1163-7.

34. Kinoshita T, Yoshida J, Ishii G, Aokage K, Hishida T, Nagai K. The differences of biological behavior based on the clinicopathological data between resectable largecell neuroendocrine carcinoma and small-cell lung carcinoma. Clin Lung Cancer 2013; 14: 535-40.

35. Shimizu K, Yoshida J, Nagai K, et al. Visceral pleural invasion is an invasive and aggressive indicator of nonsmall cell lung cancer. J Thorac Cardiovasc Surg 2005; 130: 160-5.

36. Manac'h D, Riquet M, Medioni J, Le Pimpec-Barthes F Dujon A, Danel C. Visceral pleura invasion by non-small cell lung cancer: an underrated bad prognostic factor. Ann Thorac Surg 2001; 71: 1088-93.

37. Grand B, Cazes A, Mordant P, et al. High grade neuroendocrine lung tumors: pathological characteristics, surgical management and prognostic implications. Lung Cancer 2013; 81: 404-9.

38. Fernandez FG, Battafarano RJ. Large-cell neuroendocrine carcinoma of the lung. Cancer Control 2006; 13: 270-5. 